\title{
Gradient Observability for Semilinear Hyperbolic Systems: Sectorial Approach
}

\author{
Adil Khazari, Ali Boutoulout \\ TSI Team, MACS Laboratory, Department of Mathematics \& Computer, Faculty of Sciences, \\ Moulay Ismail University, Meknes, Morocco \\ Email: adil0974@gmail.com, boutouloutali@yahoo.fr
}

Received 18 June 2014; revised 21 July 2014; accepted 1 August 2014

Copyright (C) 2014 by authors and Scientific Research Publishing Inc.

This work is licensed under the Creative Commons Attribution International License (CC BY). http://creativecommons.org/licenses/by/4.0/

cC) (7) Open Access

\begin{abstract}
The aim of this work is to study the notion of the gradient observability on a subregion $\omega$ of the evolution domain $\Omega$ for a class of semilinear hyperbolic systems. We show, under some hypothesis, that the gradient reconstruction is achieved following sectorial approach combined with fixed point techniques. The obtained results lead to an algorithm which can be implemented numerically.
\end{abstract}

\section{Keywords}

Distributed Systems, Hyperbolic Systems, Gradient Reconstruction, Regional Observability, Fixed Point, Sectorial Operator

\section{Introduction}

The regional observability is one of the most important notions of system theory, and it consists in reconstructing the initials conditions (initial state and initial speed) for hyperbolic systems only in a subregion $\omega$ of the system evolution domain $\Omega$. This concept was largely developed (see [1] [2]) for parabolic systems and for hyperbolic systems (see [3] [4]). Subsequently, the concept of regional observability was extended to the gradient observability for parabolic systems (see [5] [6]) and for hyperbolic systems (sees [7]), which consist in reconstructing directly the gradient of the initial conditions only in a critical subregion interior $\omega$ without the knowledge of the initial conditions. This concept finds its application in many real world problems.

The aim of this paper is to study the regional gradient observability of an important class of semilinear hyperbolic systems. We will focus our attention on the case where the dynamic of the system is a linear operator 
and sectorial. This approach was examined for semilinear parabolic systems to reconstruct the initial gradient state ([8]) and for semilinear hyperbolic systems to reconstruct the initial state and the initial speed. For observability problem when one is confronted to the question of reconstructing the gradient state and the gradient speed, it is important to take into account the effects of non-linearity. For example, approximate controllability of semilinear system can be obtained when the non-linearity satisfies some conditions (see [9] [10]), and the used techniques combine a variational approach to controllability problem for linear equation and fixed point method. The techniques are also based on linear infinite dimensional observability theory together with a variety of fixed point theorems.

The plan of the paper is as follows: Section 2 is devoted to the presentation of the problem of regional gradient observability of the considered system. Section 3 concerns the sectorial approach. Numerical approach is developed in the last section.

\section{Problem Statement}

Let $\Omega$ be an open bounded subset of $\operatorname{IR}^{n}(n=1,2,3)$.

For $T>0$, we denote $Q=\Omega \times] 0, T[, \Sigma=\partial \Omega \times] 0, T[$ and we consider the following semilinear hyperbolic system

$$
\begin{cases}\frac{\partial^{2} y}{\partial t^{2}}(x, t)=\mathcal{A} y(x, t)+\mathcal{N} y(x, t) & \text { in } Q \\ y(x, 0)=y^{0}(x), \frac{\partial y}{\partial t}(x, 0)=y^{1}(x) & \text { in } \Omega \\ y(\xi, t)=0 & \text { on } \Sigma\end{cases}
$$

where $\mathcal{A}$ is a second order elliptic linear operator, symmetric generating a strongly continuous semigroup $(S(t))_{t \geq 0}$ and $\mathcal{N}$ is a nonlinear operator assumed to be locally Lipshitzian.

Let $\left(y, \frac{\partial y}{\partial t}\right) \in \mathcal{F}=H_{0}^{1}(\Omega) \times H_{0}^{1}(\Omega)$ denotes the solution of system (1) (see [11]) and the function of measurements is given by the output function

$$
z(x, t)=C y(x, t)
$$

where $C$ is a linear operator from $\mathcal{F}$ to the space $I R^{q}$, and depends on the number and the nature of the considered sensors.

Let $\Phi_{m j}$ a basis of eigenfunctions of the operator $\mathcal{A}$, with Dirichlet conditions and the associated eigenvalues $\lambda_{m}$ of multiplicity $r_{m}$.

For any $\left(y_{1}, y_{2}\right) \in E=L^{2}(\Omega) \times L^{2}(\Omega)$ the semigroup $(S(t))_{t \geq 0}$ is given by

$$
\bar{S}(t)\left(\begin{array}{l}
y_{1} \\
y_{2}
\end{array}\right)=\left(\begin{array}{l}
\sum_{m}^{\infty} \sum_{j=1}^{r_{m}}\left[\left\langle y_{1}, \Phi_{m j}\right\rangle \cos \sqrt{-\lambda_{m}} t+\frac{1}{\sqrt{-\lambda_{m}}}\left\langle y_{2}, \Phi_{m j}\right\rangle \sin \sqrt{-\lambda_{m}} t\right] \Phi_{m j}(.) \\
\sum_{m}^{\infty} \sum_{j=1}^{r_{m}}\left[-\sqrt{-\lambda_{m}}\left\langle y_{1}, \Phi_{m j}\right\rangle \sin \sqrt{-\lambda_{m}} t+\left\langle y_{2}, \Phi_{m j}\right\rangle \cos \sqrt{-\lambda_{m}} t\right] \Phi_{m j}(.)
\end{array}\right)
$$

Without loss of generality we note: $y(t):=y(x ; t)$ and we associate to the system (1) the linear system

$$
\begin{cases}\frac{\partial^{2} y}{\partial t^{2}}(t)=\mathcal{A} y(t) & \text { in } Q \\ y(0)=y^{0}, \frac{\partial y}{\partial t}(0)=y^{1} & \text { in } \Omega \\ y(t)=0 & \text { on } \Sigma\end{cases}
$$


The system (3) admits a unique solution $y \in C\left(0 ; T ; H_{0}^{1}(\Omega)\right) \cap C^{1}\left(0 ; T ; L^{2}(\Omega)\right)$ (see [12]).

Let denote $\bar{y}=\left(y, \frac{\partial y}{\partial t}\right), \overline{\mathcal{A}}\left(y_{1}, y_{2}\right)=\left(y_{2}, \mathcal{A} y_{1}\right)$ for all $\left(y_{1}, y_{2}\right) \in D(\overline{\mathcal{A}})=\left(H_{0}^{1}(\Omega) \cap H^{2}(\Omega)\right) \times L^{2}(\Omega)$, $\overline{\mathcal{N}}\left(y_{1}, y_{2}\right)=\left(0, \mathcal{N} y_{1}\right)$ and $\bar{y}^{0}=\left(y^{0}, \frac{\partial y^{0}}{\partial t}=y^{1}\right)$.

The system (1) may be written as

$$
\left\{\begin{array}{l}
\frac{\partial \bar{y}}{\partial t}(t)=\overline{\mathcal{A}} \bar{y}(t)+\overline{\mathcal{N}} \bar{y}(t) \quad 0<t<T \\
\bar{y}(0)=\bar{y}^{0}
\end{array}\right.
$$

and the system (3) is equivalent to

$$
\left\{\begin{array}{l}
\frac{\partial \bar{y}}{\partial t}(t)=\overline{\mathcal{A}} \bar{y}(t) \quad 0<t<T \\
\bar{y}(0)=\bar{y}^{0}
\end{array}\right.
$$

Systems (4) and (5) are augmented with the output function

$$
\bar{z}(t)=\bar{C} \bar{y}(t) \text { with } \bar{C}=(C, 0)
$$

The system (1) can be interpreted in the mild sense as follows

$$
\bar{y}(t)=\bar{S}(t) \bar{y}^{0}+\int_{0}^{t} \bar{S}(t-\tau) \overline{\mathcal{N}} \bar{y}(\tau) \mathrm{d} \tau
$$

and the output equation can be expressed by

$$
\left.\bar{z}(t)=\overline{C S}(t) \bar{y}^{0}=\bar{K}(t) \bar{y}^{0}, t \in\right] 0, T[
$$

Let $\bar{K}$ be the observation operator defined by

$$
\begin{aligned}
& \bar{K}: \mathcal{F} \rightarrow \widetilde{\mathcal{O}}=L^{2}\left(0, T ; I R^{q}\right) \times L^{2}\left(0, T ; I R^{q}\right) \\
& \bar{Z} \mapsto \bar{C} \bar{S}(.) \bar{Z}
\end{aligned}
$$

which is linear and bounded with the adjoint $\bar{K}^{*}$.

Consider the operator $\bar{\nabla}$ given by

$$
\begin{aligned}
& \bar{\nabla}: H_{0}^{1}(\Omega) \times H_{0}^{1}(\Omega) \rightarrow\left(L^{2}(\Omega)\right)^{n} \times\left(L^{2}(\Omega)\right)^{n} \\
& \left(y_{1}, y_{2}\right) \mapsto \bar{\nabla}\left(y_{1}, y_{2}\right)=\left(\nabla y_{1}, \nabla y_{2}\right)
\end{aligned}
$$

where

$$
\begin{aligned}
& \nabla: H_{0}^{1}(\Omega) \rightarrow\left(L^{2}(\Omega)\right)^{n} \\
& y \mapsto \nabla y=\left(\frac{\partial y}{\partial x_{1}}, \cdots, \frac{\partial y}{\partial x_{n}}\right)
\end{aligned}
$$

$\bar{\nabla}^{*}$ is the adjoint of $\bar{\nabla}$.

The initial condition $\bar{y}^{0}$ and $\bar{\nabla} \bar{y}^{0}$ its gradient are assumed to be unknown.

For $\omega \subset \Omega$ an open subregion of $\Omega$ and of positive Lebesgue measure, let $\bar{\chi}_{\omega}$ be the restriction operator defined by

$$
\begin{aligned}
& \bar{\chi}_{\omega}:\left(L^{2}(\Omega)\right)^{n} \times\left(L^{2}(\Omega)\right)^{n} \rightarrow\left(L^{2}(\omega)\right)^{n} \times\left(L^{2}(\omega)\right)^{n} \\
& \left(y_{1}, y_{2}\right) \mapsto \bar{\chi}_{\omega}\left(y_{1}, y_{2}\right)=\left(\chi_{\omega} y_{1}, \chi_{\omega} y_{2}\right)
\end{aligned}
$$

where 


$$
\begin{aligned}
& \chi_{\omega}:\left(L^{2}(\Omega)\right)^{n} \rightarrow\left(L^{2}(\omega)\right)^{n} \\
& \xi \mapsto \bar{\chi}_{\omega} \xi
\end{aligned}
$$

$\bar{\chi}_{\omega}^{*}$. (resp. $\left.\chi_{\omega}^{*}\right)$ is the adjoint of $\bar{\chi}_{\omega}$ (resp. $\chi_{\omega}$ ), and we consider the operator

$$
\bar{H}=\bar{\chi}_{\omega} \bar{\nabla} \bar{K}^{*}
$$

Let $\bar{\nabla} \bar{y}^{0}=\left(\nabla y^{0}, \nabla y^{1}\right)$ be the gradient of the initial condition $\bar{y}^{0}=\left(y^{0}, y^{1}\right)$, we have

$$
\bar{\nabla} \bar{y}^{0}= \begin{cases}\bar{y}_{1}^{0} & \text { in } \omega \\ \bar{y}_{2}^{0} & \text { in } \Omega \backslash \omega\end{cases}
$$

where $\bar{y}_{1}^{0}=\left(y_{1}^{0}, \frac{\partial y_{1}^{0}}{\partial t}=y_{1}^{1}\right), \quad \bar{y}_{2}^{0}=\left(y_{2}^{0}, \frac{\partial y_{2}^{0}}{\partial t}=y_{2}^{1}\right)$ and

$$
\nabla y^{0}=\left\{\begin{array}{ll}
y_{1}^{0} & \text { in } \omega \\
y_{2}^{0} & \text { in } \Omega \backslash \omega
\end{array}, \quad \nabla y^{1}= \begin{cases}y_{1}^{1} & \text { in } \omega \\
y_{2}^{1} & \text { in } \Omega \backslash \omega\end{cases}\right.
$$

\section{Definition 1.}

The System (3)-(2) is said to be exactly (respectively. weakly) $G$-observable in $\omega$ if $\operatorname{Im}(\bar{H})=\left(L^{2}(\omega)\right)^{n}$ (respectively. $\overline{\operatorname{Im}(\bar{H})}=\left(L^{2}(\omega)\right)^{n}$.

\section{Definition 2.}

The semilinear system (1) augmented with output (2) is said to be gradient observable in $\omega$ ( $G$-observable in $\omega$ ) if we can reconstruct the gradient of its state and the gradient of its speed in a subregion $\omega$ of $\Omega$ at any time $t$.

The study of regional gradient observability leads to solving the following problem:

\section{Problem 1.}

Given the semilinear system (1) and output (2) on $[0, T]$, is it possible to reconstruct $\bar{\nabla} \bar{y}^{0}=\left(\nabla y^{0}, \nabla y^{1}\right)$ which is the gradient of initial state and the gradient of initial speed of (1) in $\omega$ ?

Let's consider $\mathcal{X}=L^{2}(0, T ; E)$ and we define, for $\left.t \in\right] 0, T[$, the operator $L():. \mathcal{X} \rightarrow \mathcal{X}$ by

$$
\left.L(t) \bar{x}(.)=\int_{0}^{t} \bar{S}(t-s) \bar{x}(s) \mathrm{d} s, \quad \forall t \in\right] 0, T\left[\text { and } \bar{x}=\left[\begin{array}{l}
x_{1} \\
x_{2}
\end{array}\right]\right.
$$

then we have the following results:

\section{Proposition 1.}

If the system (3) is weakly $G$-observable, then the solution $\bar{y}($.$) of the system (6) is a fixed point of the$ mapping $\Phi():. \mathcal{X} \rightarrow \mathcal{X}$ defined by:

$$
\Phi(t)(\bar{y}(.))=\bar{S}(t) \bar{\nabla}^{*} \bar{\chi}_{\omega}^{*}\left[\bar{H}^{*}\right]^{\dagger}\left(\bar{z}(.)-\bar{C} L(.) \overline{\mathcal{N}} \bar{y}(.)-\bar{K}(.) \hat{y}_{2}^{0}\right)+\bar{S}(t) \hat{y}_{2}^{0}+L(t) \overline{\mathcal{N}} \bar{y}(.)
$$

where $\left[\bar{H}^{*}\right]^{\dagger}$ is the pseudo inverse of the operator $\bar{H}^{*}$ and $\hat{y}_{2}^{0} \in \mathcal{F}$ such that

$$
\bar{y}^{0}=\bar{\nabla}^{*} \bar{\chi}_{\omega}^{*} \bar{y}_{1}^{0}+\hat{y}_{2}^{0}
$$

where $\hat{y}_{2}^{0}$ is the residual part.

\section{Proof}

The solution of the system (4) can be expressed by $\bar{y}(t)=\bar{S}(t) \bar{y}^{0}+L(t) \overline{\mathcal{N}} \bar{y}($.$) thus,$ $\overline{C y}(t)=\bar{C} \bar{S}(t) \bar{y}^{0}+\bar{C} L(t) \overline{\mathcal{N}} \bar{y}($.$) so we have$

$$
\bar{K}(.) \bar{y}^{0}=\bar{z}-\bar{C} L(.) \overline{\mathcal{N}} \bar{y}(.)
$$

where $\bar{Z}$ is the output function which allows information about the considered system.

Using the second decomposition of initial condition we obtain $\bar{K}().\left(\bar{\nabla}^{*} \bar{\chi}_{\omega}^{*} \bar{y}_{1}^{0}+\hat{y}_{2}^{0}\right)=\bar{z}-\bar{C} L(.) \overline{\mathcal{N}} \bar{y}$ which is equivalent to $\bar{H}^{*} \bar{y}_{1}^{0}+\bar{K}(.) \hat{y}_{2}^{0}=\bar{z}-\bar{C} L(.) \overline{\mathcal{N}} \bar{y}$.

If the linear part of the system (1) is weakly $G$-observable in $\omega$, then we have 


$$
\bar{y}_{1}^{0}=\left[\bar{H}^{*}\right]^{\dagger}\left(\bar{z}-\bar{C} L(.) \overline{\mathcal{N}} \bar{y}-\bar{K}(.) \hat{y}_{2}^{0}\right)
$$

where $\left[\bar{H}^{*}\right]^{\dagger}=\left[\bar{H} \bar{H}^{*}\right]^{-1} \bar{H}$ is the pseudo inverse of the operator $\bar{H}^{*}$.

Finally, solution of problem of $G$-observability in $\omega$ is a fixed point of the following function: $\Phi():. \mathcal{X} \rightarrow \mathcal{X}$ define by:

$$
\Phi(t)(\bar{y}(.))=\bar{S}(t) \bar{\nabla}^{*} \bar{\chi}_{\omega}^{*}\left[\bar{H}^{*}\right]^{\dagger}\left(\bar{z}(.)-\bar{C} L(.) \overline{\mathcal{N}} \bar{y}(.)-\bar{K}(.) \hat{y}_{2}^{0}\right)+\bar{S}(t) \hat{y}_{2}^{0}+L(t) \overline{\mathcal{N}} \bar{y}(.)=\bar{y}(t)
$$

Proposition 2.

If $\operatorname{Im} \bar{H}^{*}$ is closed in $L^{2}\left(0, T ; I R^{q}\right)$ and if the function (9) has a unique fixed point $\bar{y}($.$) such that$

$$
\left[\bar{z}(.)-\bar{C} L(.) \overline{\mathcal{N}} \bar{y}(.)-\bar{K}(.) \hat{y}_{2}^{0}\right] \in \operatorname{Im} \bar{H}^{*}
$$

then $\left.\bar{\nabla} \bar{y}(0)\right|_{\omega}=\left.\bar{\nabla} \bar{y}^{0}\right|_{\omega}$ is the initial gradient to be observed in $\omega$ of system (4).

Proof

Let $\bar{y}($.$) a fixed point of equation (9), then$

$$
\bar{C} \bar{y}(.)=\left[\bar{H}^{*}\right]\left[\bar{H}^{*}\right]^{\dagger}\left(\bar{z}(.)-\bar{C} L(.) \overline{\mathcal{N}} \bar{y}(.)-\bar{K}(.) \hat{y}_{2}^{0}\right)+\bar{C} \bar{S}(t) \hat{y}_{2}^{0}+\bar{C} L(t) \overline{\mathcal{N}} \bar{y}(.)=\bar{y}(t)
$$

But the operator $\left[\bar{H}^{*}\right]\left[\bar{H}^{*}\right]^{\dagger}$ is the orthogonal projection of $L^{2}\left(0, T ; \operatorname{IR} R^{q}\right)$ in $\operatorname{Im} \bar{H}^{*}$ and $\bar{y}($.$) satisfy$ condition (10), then $\bar{C} \bar{y}()=.\bar{z}($.$) .$

Finally $\left.\bar{\nabla} \bar{y}(0)\right|_{\omega}=\left.\bar{\nabla} \bar{y}^{0}\right|_{\omega}=\bar{y}_{1}^{0}=\left[\bar{H}^{*}\right]^{\dagger}\left(\bar{z}()-.\bar{C} L(.) \overline{\mathcal{N}} \bar{y}()-.\bar{K}(.) \hat{y}_{2}^{0}\right)$

which is the initial gradient to be observed in $\omega$ of system (4).

\section{Sectorial Approach}

In this section, we study Problem 1 under some supplementary hypothesis on $\mathcal{A}$ and the nonlinear operator $\mathcal{N}$.

With the same notations as in the previous case, we reconsider the semilinear system described by the equations (4) and (6) where one supposed that the operator $\overline{\mathcal{A}}$ generates an analytic semigroup $(\bar{S}(t))_{t \geq 0}$ in the state space $E$.

Let's consider $\overline{\mathcal{A}}_{1}=\overline{\mathcal{A}}+a I$ such that $\operatorname{Re}\left(\sigma\left(\overline{\mathcal{A}}_{1}\right)\right)>\delta>0$ with $a$ is a positive real number and $\operatorname{Re}\left(\sigma\left(\overline{\mathcal{A}}_{1}\right)\right)$ denotes the real part of spectrum of $\overline{\mathcal{A}}_{1}$. Then for $0 \leq \alpha<1$, we define the fractional power $\left(\overline{\mathcal{A}}_{1}\right)^{\alpha}$ as a closed operator with domain $E^{\alpha}=D\left(\overline{\mathcal{A}}_{1}^{\alpha}\right)$ which is a dense Banach space on $E$ endowed with the graph norm

$$
\|\cdot\|_{E^{\alpha}}=\left\|\overline{\mathcal{A}}_{1}^{\alpha}(.)\right\|_{E}
$$

and consider $V=\operatorname{Im}\left(\bar{\chi}_{\omega} \bar{\nabla} \bar{K}^{*}\right)$.

We consider Problem 1 in $V$ endowed with the norm

$$
\|\cdot\|_{V}=\left\|\bar{H}^{*}(.)\right\|_{\tilde{o}}
$$

We have

$$
\|\bar{S}(t)\|_{\mathcal{L}\left(E, E^{\alpha}\right)} \leq c t^{-\alpha} \exp (a-\alpha) t=g_{1}(t)
$$

where $c$ is a constant. For more details, see ([2] [6] [13])

For $r, s>1$, assume that

$$
\left\{\begin{array}{l}
\frac{1}{r}+\frac{1}{s}=1 \\
g_{1} \in L^{r}(0, T)
\end{array}\right.
$$

And the operator $\overline{\mathcal{N}}: L^{r}\left(0, T ; E^{\alpha}\right) \rightarrow L^{s}(0, T ; E)$ is well defined and satisfies the following conditions: 


$$
\left\{\begin{array}{l}
\|\overline{\mathcal{N}} x-\overline{\mathcal{N}} y\|_{L^{5}(0, T ; E)} \leq k(\|x\|,\|y\|)\|x-y\|_{L^{r}\left(0, T ; E^{\alpha}\right)} \forall x, y \in L^{r}\left(0, T ; E^{\alpha}\right) \\
\overline{\mathcal{N}}(0)=0 \quad \text { with } k: I R^{+} \times I R^{+} \rightarrow I R^{+} \\
\text {such that } \lim _{\left(\theta_{1}, \theta_{2}\right) \rightarrow(0,0)} k\left(\theta_{1}, \theta_{2}\right)=0
\end{array}\right.
$$

Those hypothesis are verified by much important class of semi linear hyperbolic systems. For example the equation governing the flow of neutrons in a nuclear reactor

$$
\begin{cases}\frac{\partial y}{\partial t}(t)-k \frac{\partial^{2} y}{\partial t^{2}}(t)-a y(t)=-b y^{2}(t) & \\ y(t)=0 & \text { in }[0,1] \\ y(0)=0 & \end{cases}
$$

which $y(t) \in L^{2}([0,1]), 0 \leq t \leq T, k, a, b>0$.

The operators $\mathcal{A}$ and $\mathcal{N}$ corresponding are

$$
\begin{gathered}
\mathcal{A}: H_{0}^{1}([0,1]) \cap H^{2}([0,1]) \rightarrow L^{2}([0,1]) \mathcal{N}: H_{0}^{1}([0,1]) \rightarrow L^{2}([0,1]) \\
y \mapsto \frac{1}{k} \frac{\partial y}{\partial t}-\frac{a}{k} y \quad ; \quad y \mapsto \frac{b y^{2}}{k}
\end{gathered}
$$

The assumption is satisfied with $r=2, s=1$ and $k\left(\theta_{1}, \theta_{2}\right)=\frac{b}{k}\left(\theta_{1}+\theta_{2}\right)$.

Various examples are given and discussed in ([13] [14]).

We show that exists a set of admissible initial gradient state and admissible initial gradient speed, admissible in the sense that system (3) be weakly $G$-observable.

Let's consider $\varphi\left(\bar{y}_{1}^{0},.\right): L^{r}\left(0, T ; E^{\alpha}\right) \rightarrow L^{r}\left(0, T ; E^{\alpha}\right)$ given by

$$
\varphi\left(\bar{y}_{1}^{0}, \bar{y}\right)(t)=\bar{S}(t) \bar{\nabla}^{*} \bar{\chi}_{\omega}^{*} \bar{y}_{1}^{0}+\bar{S}(t) \hat{y}_{2}^{0}+L(t) \bar{N} \bar{y} \quad t \in[0, T]
$$

where $\bar{y}_{1}^{0}$ is the restriction in $\omega$ and $\hat{y}_{2}^{0}$ is the residual part in $\Omega \backslash \omega$ of the initial gradient condition $\bar{\nabla} \bar{y}^{0}$. We assume that

$$
\left\|\bar{S}(t) \bar{\nabla}^{*} \bar{\chi}_{\omega}^{*}\right\|_{\mathcal{L}\left(V, E^{\alpha}\right)} \leq g_{2}(t) \text { with } g_{2} \in L^{2}(0, T)
$$

then we have the following result.

Proposition 3.

Suppose that system (3) is weakly $G$-observable in $\omega$, and (12), (13) and (14) satisfied, then the following assertion hold:

- There exist $a_{1}>0$ and $m>0$ such that for all $\bar{y}_{1}^{0} \in B(0, m) \subset V$ the function $\varphi\left(\bar{y}_{1}^{0},.\right)$ has a unique fixed point $\bar{y}$ in the ball $B\left(0, a_{1}\right) \subset L^{r}\left(0, T ; E^{\alpha}\right)$ solution of the system (4).

- There exist $m=m\left(a_{1}\right)$ and $m_{1}=m_{1}\left(a_{1}\right)$ such that $\left\|\hat{y}_{2}^{0}\right\|_{E} \leq m_{1}$ the mapping $f$ is lipschitzian where

$$
\begin{gathered}
f: B(0, m) \rightarrow B\left(0, a_{1}\right) \\
\bar{y}_{1}^{0} \mapsto \bar{y}
\end{gathered}
$$

\section{Proof}

- Since $\lim _{\left(\theta_{1}, \theta_{2}\right) \rightarrow(0,0)} k\left(\theta_{1}, \theta_{2}\right)=0$, then there exists $\left.a_{1} \in\right] 0,1\left[\right.$ such that $\alpha_{1}=T^{\frac{1}{r}}\left\|g_{1}\right\|_{L^{r}(0, T)} \sup _{\theta_{1}, \theta_{2} \leq a_{1}} k\left(\theta_{1}, \theta_{2}\right)<1$ and we have $\left.\alpha_{1} \in\right] 0,1[$.

Let us consider $\bar{y}$ and $\bar{x}$ in $B\left(0, a_{1}\right) \subset L^{r}\left(0, T ; E^{\alpha}\right)$ and $\bar{y}_{1}^{0} \in V$ we have

$$
\left\|\varphi\left(\bar{y}_{1}^{0}, \bar{y}\right)-\varphi\left(\bar{y}_{1}^{0}, \bar{x}\right)\right\|_{L^{r}\left(0, T ; E^{\alpha}\right)}=\|L(.)(\bar{N} \bar{y}-\bar{N} \bar{x})\|_{L^{r}\left(0, T ; E^{\alpha}\right)}=\left(\int_{0}^{T}\|L(t)(\bar{N} \bar{y}-\bar{N} \bar{x})\|_{E^{\alpha}}^{r} \mathrm{~d} t\right)^{\frac{1}{r}}
$$


A. Khazari, A. Boutoulout

where

$$
\begin{aligned}
\|L(t)(\bar{N} \bar{y}-\bar{N} \bar{x})\|_{E^{\alpha}} & =\left\|\int_{0}^{t} \bar{S}(t-\tau)(\bar{N} \bar{y}(\tau)-\bar{N} \bar{x}(\tau)) \mathrm{d} \tau\right\|_{E^{\alpha}} \\
& \leq \int_{0}^{T}\|\bar{S}(t-\tau)(\bar{N} \bar{y}(\tau)-\bar{N} \bar{x}(\tau))\|_{E^{\alpha}} \mathrm{d} \tau \\
& \leq \int_{0}^{T}\|\bar{S}(t-\tau)\|_{\mathcal{L}\left(E, E^{\alpha}\right)}\|(\bar{N} \bar{y}(\tau)-\bar{N} \bar{x}(\tau))\|_{E} \mathrm{~d} \tau \\
& \leq \int_{0}^{T} g_{1}(t)\|(\bar{N} \bar{y}(\tau)-\bar{N} \bar{x}(\tau))\|_{E} \mathrm{~d} \tau
\end{aligned}
$$

Using Holder's inequality we take $\|L(t)(\bar{N} \bar{y}-\bar{N} \bar{x})\|_{E^{\alpha}}^{r} \leq\left\|g_{1}(t)\right\|_{L^{r}(0, T)}^{r}\|\bar{N} \bar{y}-\bar{N} \bar{x}\|_{L^{5}(0, T ; E)}^{r}$ and using (13), we have

$$
\begin{aligned}
\left\|\varphi\left(\bar{y}_{1}^{0}, \bar{y}\right)-\varphi\left(\bar{y}_{1}^{0}, \bar{x}\right)\right\|_{L^{r}\left(0, T ; E^{\alpha}\right)} & \leq\left(\int_{0}^{T}\left\|g_{1}(t)\right\|_{L^{r}(0, T)}^{r}\|\bar{N} \bar{y}-\bar{N} \bar{x}\|_{L^{s}(0, T ; E)}^{r} \mathrm{~d} t\right)^{\frac{1}{r}} \\
& \leq T^{\frac{1}{r}}\left\|g_{1}(t)\right\|_{L^{r}(0, T)}\|\bar{N} \bar{y}-\bar{N} \bar{x}\|_{L^{5}(0, T ; E)} \\
& \leq T^{\frac{1}{r}}\left\|g_{1}\right\|_{L^{r}(0, T)} k(\|\bar{y}\|,\|\bar{x}\|)\|\bar{y}-\bar{x}\|_{L^{r}\left(0, T ; E^{\alpha}\right)} \\
& \leq T^{\frac{1}{r}}\left\|g_{1}\right\|_{L^{r}(0, T)} \sup _{\|\bar{y}\|\|\| \| \leq a_{1}} k(\|\bar{y}\|,\|\bar{x}\|)\|\bar{y}-\bar{x}\|_{L^{r}\left(0, T ; E^{\alpha}\right)} \\
& \leq \alpha_{1}\|\bar{y}-\bar{x}\|_{L^{r}\left(0, T ; E^{\alpha}\right)}
\end{aligned}
$$

On the other hand, we have

$$
\begin{aligned}
\left\|\varphi\left(\bar{y}_{1}^{0}, \bar{y}\right)\right\|_{L^{r}\left(0, T ; E^{\alpha}\right)} & =\left\|\bar{S}(.) \bar{\nabla}^{*} \bar{\chi}_{\omega}^{*} \bar{y}_{1}^{0}+\bar{S}(.) \hat{y}_{2}^{0}+L(.) \bar{N} \bar{y}\right\|_{L^{r}\left(0, T ; E^{\alpha}\right)} \\
& \leq\left\|\bar{S}(.) \bar{\nabla}^{*} \bar{\chi}_{\omega}^{*} \bar{y}_{1}^{0}\right\|_{L^{r}\left(0, T ; E^{\alpha}\right)}+\left\|\bar{S}(.) \hat{y}_{2}^{0}\right\|_{L^{r}\left(0, T ; E^{\alpha}\right)}+\|L(.) \bar{N} \bar{y}\|_{L^{r}\left(0, T ; E^{\alpha}\right)} \\
& \leq\left(\int_{0}^{T}\left\|\bar{S}(t) \bar{\nabla}^{*} \bar{\chi}_{\omega}^{*} \bar{y}_{1}^{0}\right\|_{E^{\alpha}}^{r} \mathrm{~d} t\right)^{\frac{1}{r}}+\left(\int_{0}^{T}\left\|\bar{S}(t) \hat{y}_{2}^{0}\right\|_{E^{\alpha}}^{r} \mathrm{~d} t\right)^{\frac{1}{r}}+\left(\int_{0}^{T}\|L(t) \bar{N} \bar{y}\|_{E^{\alpha}}^{r} \mathrm{~d} t\right)^{\frac{1}{r}}
\end{aligned}
$$

but we have

$$
\| \bar{S}(t)) \bar{\nabla}^{*} \bar{\chi}_{\omega}^{*} \bar{y}_{1}^{0}\left\|_{E^{\alpha}} \leq\right\| \bar{S}(t) \bar{\nabla}^{*} \bar{\chi}_{\omega}^{*}\left\|_{\mathcal{L}\left(v, E^{\alpha}\right)}\right\| \bar{y}_{1}^{0}\left\|_{V} \leq g_{2}(t)\right\| \bar{y}_{1}^{0} \|_{V}
$$

and

$$
\left\|\bar{S}(t) \hat{y}_{2}^{0}\right\|_{E^{\alpha}} \leq\|\bar{S}(t)\|_{E^{\alpha}}\left\|\hat{y}_{2}^{0}\right\|_{E} \leq g_{1}(t)\left\|\hat{y}_{2}^{0}\right\|_{E}
$$

and using Holder's inequality we obtain

$$
\begin{aligned}
\|L(t) \bar{N} \bar{y}\|_{E^{\alpha}} & =\left\|\int_{0}^{T} \bar{S}(t-\tau) \bar{N} \bar{y}(\tau) \mathrm{d} \tau\right\|_{E^{\alpha}} \leq \int_{0}^{T}\|\bar{S}(t-\tau)\|_{\mathcal{L}\left(E, E^{\alpha}\right)}\|\bar{N} \bar{y}(\tau)\|_{E} \mathrm{~d} \tau \\
& \leq\left\|g_{1}(t)\right\|_{L^{r}(0, T)}\|\bar{N} \bar{y}\|_{L^{s}\left(0, T ; E^{\alpha}\right)} \\
& \leq\left\|g_{1}(t)\right\|_{L^{r}(0, T)} k(\|\bar{y}\|, 0)\|\bar{y}\|_{L^{r}\left(0, T ; E^{\alpha}\right)} \\
& \leq\left\|g_{1}(t)\right\|_{L^{r}(0, T)} \sup k\left(\theta \leq a_{1}\right.
\end{aligned}
$$

176 
then we have

$$
\left\|\bar{S}(.) \bar{\nabla}^{*} \bar{\chi}_{\omega}^{*} \bar{y}_{1}^{0}\right\|_{L^{r}\left(0, T ; E^{\alpha}\right)}=\left(\int_{0}^{T}\left\|\bar{S}(t) \bar{\nabla}^{*} \bar{\chi}_{\omega}^{*} \bar{y}_{1}^{0}\right\|_{E^{\alpha}}^{r} \mathrm{~d} t\right)^{\frac{1}{r}} \leq\left(\int_{0}^{T} g_{2}^{r}(t)\left\|\bar{y}_{1}^{0}\right\|_{V}^{r} \mathrm{~d} t\right)^{\frac{1}{r}} \leq\left\|g_{2}(t)\right\|_{L^{r}(0, T)}\left\|\bar{y}_{1}^{0}\right\|_{V}
$$

and

$$
\left\|\bar{S}(.) \hat{y}_{2}^{0}\right\|_{L^{r}\left(0, T ; E^{\alpha}\right)}=\left(\int_{0}^{T}\left\|\bar{S}(t) \hat{y}_{2}^{0}\right\|_{E^{\alpha}}^{r} \mathrm{~d} t\right)^{\frac{1}{r}} \leq\left(\int_{0}^{T} g_{1}^{r}(t)\left\|\hat{y}_{2}^{0}\right\|_{E}^{r} \mathrm{~d} t\right)^{\frac{1}{r}} \leq\left\|g_{1}(t)\right\|_{L^{r}(0, T)}\left\|\hat{y}_{2}^{0}\right\|_{E}
$$

or

$$
\begin{aligned}
\|L(.) \bar{N} \bar{y}\|_{L^{r}\left(0, T ; E^{\alpha}\right)} & =\left(\int_{0}^{T}\|L(t) \bar{N} \bar{y}\|_{E^{\alpha}}^{r} \mathrm{~d} t\right)^{\frac{1}{r}} \\
& \leq T^{\frac{1}{r}}\left\|g_{1}(t)\right\|_{L^{r}(0, T)} \sup _{\theta \leq a_{1}} k(\theta, 0)\|\bar{y}\|_{L^{r}\left(0, T ; E^{\alpha}\right)} \\
& \leq \alpha_{2}\|\bar{y}\|_{\mathcal{L}\left(0, T ; E^{\alpha}\right)}
\end{aligned}
$$

where $\alpha_{2}=T^{\frac{1}{r}}\left\|g_{1}(t)\right\|_{L^{r}(0, T)} \sup _{\theta \leq a_{1}} k(\theta, 0)$
Finally

$$
\left\|\varphi\left(\bar{y}_{1}^{0}, \bar{y}\right)\right\|_{L^{r}\left(0, T ; E^{\alpha}\right)} \leq\left\|g_{2}(t)\right\|_{L^{r}(0, T)}\left\|\bar{y}_{1}^{0}\right\|_{V}+\left\|g_{1}(t)\right\|_{L^{r}(0, T)}\left\|\hat{y}_{2}^{0}\right\|_{E}+\alpha_{2}\|\bar{y}\|_{L^{r}\left(0, T ; E^{\alpha}\right)}
$$

Let's consider $m=m\left(a_{1}\right)=\frac{a_{1}\left(1-\alpha_{2}\right)-\left\|\hat{y}_{2}^{0}\right\|_{E}\left\|g_{1}(t)\right\|_{L^{r}(0, T)}}{\left\|g_{2}(t)\right\|_{L^{r}(0, T)}}$ and $m_{1}=m_{1}\left(a_{1}\right)=\frac{a_{1}\left(1-\alpha_{2}\right)}{\left\|g_{1}(t)\right\|_{L^{r}(0, T)}} \quad 1-\alpha_{2}>0$, then $m>0$.

It is sufficient to take $\left\|\hat{y}_{2}^{0}\right\|_{E}<m_{1}$ and $\left\|\bar{y}_{1}^{0}\right\|_{V}<m$, then for all $\bar{y} \in B\left(0, a_{1}\right)$ we have $\varphi\left(\bar{y}_{1}^{0}, \bar{y}\right) \in B\left(0, a_{1}\right)$.

Let $\bar{y}_{1}$ and $\bar{x}_{1}$ be the solution of system (4) corresponding respectively to the initial gradient in $\omega$, we suppose that we have the same residual part $\left(\bar{y}_{2}^{0}=\bar{x}_{2}^{0}\right)$, then for $\bar{y}_{1}^{0}, \bar{x}_{1}^{0} \in B(0, m)$ we have

$$
\begin{aligned}
\left\|f\left(\bar{y}_{1}^{0}\right)-f\left(\bar{x}_{1}^{0}\right)\right\|_{L^{r}\left(0, T ; E^{\alpha}\right)} & =\left\|\varphi\left(\bar{y}_{1}^{0}, \bar{y}\right)-\varphi\left(\bar{x}_{1}^{0}, \bar{x}\right)\right\|_{L^{r}\left(0, T ; E^{\alpha}\right)}=\|\bar{y}-\bar{x}\|_{L^{r}\left(0, T ; E^{\alpha}\right)} \\
& =\left\|\varphi\left(\bar{y}_{1}^{0}, \bar{y}\right)-\varphi\left(\bar{x}_{1}^{0}, \bar{x}\right)\right\|_{L^{r}\left(0, T ; E^{\alpha}\right)} \\
& =\left\|\bar{S}(.) \bar{\nabla}^{*} \bar{\chi}_{\omega}^{*}\left(\bar{y}_{1}^{0}-\bar{x}_{1}^{0}\right)+L(.)(\bar{N} \bar{y}-\bar{N} \bar{x})\right\|_{L^{r}\left(0, T ; E^{\alpha}\right)} \\
& \leq\left\|\bar{y}_{1}^{0}-\bar{x}_{1}^{0}\right\|_{V}+\|L(.)(\bar{N} \bar{y}-\bar{N} \bar{x})\|_{L^{r}\left(0, T ; E^{\alpha}\right)}
\end{aligned}
$$

but we have

$$
\begin{aligned}
\|L(.)(\bar{N} \bar{y}-\bar{N} \bar{x})\|_{L^{r}\left(0, T ; E^{\alpha}\right)} & \leq T^{\frac{1}{r}}\left\|g_{1}\right\|_{L^{r}(0, T)}\|\bar{N} \bar{y}-\bar{N} \bar{x}\|_{L^{s}(0, T ; E)} \\
& \leq T^{\frac{1}{r}}\left\|g_{1}\right\|_{L^{r}(0, T)} \sup _{\theta_{1}, \theta_{2} \leq a_{1}} K\left(\theta_{1}, \theta_{2}\right)\|\bar{y}-\bar{x}\|_{L^{r}\left(0, T ; E^{\alpha}\right)} \\
& \leq \alpha_{1}\|\bar{y}-\bar{x}\|_{L^{r}\left(0, T ; E^{\alpha}\right)}
\end{aligned}
$$

and we deduce that

$$
\left\|f\left(\bar{y}_{1}^{0}\right)-f\left(\bar{x}_{1}^{0}\right)\right\|_{L^{r}\left(0, T ; E^{\alpha}\right)} \leq \frac{\left\|g_{2}\right\|_{L^{r}(0, T)}}{1-\alpha_{1}}\left\|\bar{y}_{1}^{0}-\bar{x}_{1}^{0}\right\|_{V}
$$


Finally $f$ is lipschitzian in $B(0, m)$.

\section{Remark 1.}

The given results show that there exists a set of admissible gradient initial state. If the gradient initial state is taken in $B(0, m)$, with a bounded residual part then the system (4) has only one solution in $B\left(0, a_{1}\right)$.

Here we show that if measurements are in $B(0, \rho)$, with $\rho$ is suitably chosen then the gradient initial state can be obtained as a solution of a fixed point problem.

Let us consider the mapping

$$
\varphi_{1}\left(\bar{z}, \bar{y}_{1}^{0}\right)=\left(\bar{K} \bar{\nabla}^{*} \bar{\chi}_{\omega}^{*}\right)^{\dagger}\left(\bar{z}-\bar{C} \bar{S}(.) \hat{y}_{2}^{0}-\bar{C} L(.) \bar{N} f\left(\bar{y}_{1}^{0}\right)\right)
$$

and assume that $\forall \bar{y}_{1}^{0} \in V, \bar{C} L(.) \bar{N} f\left(\bar{y}_{1}^{0}\right) \in \operatorname{Im}\left(\bar{K} \bar{\nabla}^{*} \bar{\chi}_{\omega}^{*}\right)$.

Then we have the following result.

\section{Proposition 4.}

Assume that

$$
\begin{gathered}
\exists c_{1}>0 \quad \text { such that }\|\bar{C} L(.) \bar{y}\|_{\tilde{O}} \leq c_{1}\|\bar{y}\|_{L^{s}(0, T ; E)} \quad \forall \bar{y} \in L^{s}(0, T ; E) \\
\text { and } \exists c_{2}>0 \quad \text { such that }\|\overline{C S}(.) \bar{y}\|_{\tilde{O}} \leq c_{2}\|\bar{y}\|_{E} \quad \forall \bar{y} \in E
\end{gathered}
$$

and if the linear system(3)is weakly $G$-observable in $\omega$ and (13) holds, then there exists $a_{2}>0$ and $\rho=\rho\left(a_{2}\right)>0$, such that for all $\bar{Z} \in B(0, \rho) \subset \tilde{O}$, the function (16) admit a unique fixed point in $B(0, m)$ which corresponds to the gradient initial condition $\bar{y}_{0}^{1}$ observed in $\omega$. Furthermore, the function

$$
\begin{gathered}
h: B(0, \rho) \rightarrow B(0, m) \\
\bar{z} \mapsto \bar{y}_{1}^{0}
\end{gathered}
$$

is lipschitzian.

Proof

Let us consider $\bar{y}_{1}^{0}$ and $\bar{x}_{1}^{0}$ in $B(0, m) \subset V$, using (11), (13), (15) and (17) we have

$$
\begin{aligned}
\left\|\varphi_{1}\left(\bar{z}, \bar{y}_{1}^{0}\right)-\varphi_{1}\left(\bar{z}, \bar{x}_{1}^{0}\right)\right\|_{V} & =\left\|\left(\bar{K} \bar{\nabla}^{*} \bar{\chi}_{\omega}^{*}\right)^{\dagger}\left(\bar{C} L(.)\left(\bar{N} f\left(\bar{y}_{1}^{0}\right)-\bar{N} f\left(\bar{x}_{1}^{0}\right)\right)\right)\right\|_{V} \\
& =\left\|\left(\bar{K} \bar{\nabla}^{*} \bar{\chi}_{\omega}^{*}\right)\left(\bar{K} \bar{\nabla}^{*} \bar{\chi}_{\omega}^{*}\right)^{\dagger}\left(\bar{C} L(.)\left(\bar{N} f\left(\bar{y}_{1}^{0}\right)-\bar{N} f\left(\bar{x}_{1}^{0}\right)\right)\right)\right\|_{\tilde{o}} \\
& \leq c_{1}\left\|\bar{N} f\left(\bar{y}_{1}^{0}\right)-\bar{N} f\left(\bar{x}_{1}^{0}\right)\right\|_{L^{s}(0, T ; E)} \\
& \leq c_{1} k\left(\left\|f\left(\bar{y}_{1}^{0}\right)\right\|,\left\|f\left(\bar{x}_{1}^{0}\right)\right\|\right)\left\|f\left(\bar{y}_{1}^{0}\right)-f\left(\bar{x}_{1}^{0}\right)\right\|_{L^{r}\left(0, T ; E^{\alpha}\right)}
\end{aligned}
$$

or $\lim _{\left(\theta_{1}, \theta_{2}\right) \rightarrow(0,0)} k\left(\theta_{1}, \theta_{2}\right)=0$, then there exists $a_{2}>0$ such that $\alpha_{3}=c_{1} \sup _{\theta_{1}, \theta_{2} \leq a_{2}} K\left(\theta_{1}, \theta_{2}\right) \frac{\left\|g_{1}\right\|_{L^{r}(0, T)}}{1-\alpha_{1}}<1$ and we have $\left.\alpha_{3} \in\right] 0,1\left[\right.$. Then we obtain $\left\|\varphi_{1}\left(\bar{z}, \bar{y}_{1}^{0}\right)-\varphi_{1}\left(\bar{z}, \bar{x}_{1}^{0}\right)\right\|_{V} \leq \alpha_{3}\left\|\bar{y}_{1}^{0}-\bar{x}_{1}^{0}\right\|_{V}$.

On the other hand, using the inequality (13), (17) and (18), we have

$$
\begin{aligned}
\left\|\varphi_{1}\left(\bar{z}, \bar{y}_{1}^{0}\right)\right\|_{V} & =\left\|\bar{z}-\bar{C} \bar{S}(.) \hat{y}_{2}^{0}-\bar{C} L(.) \bar{N} f\left(\bar{y}_{1}^{0}\right)\right\|_{\tilde{O}} \\
& \leq\|\bar{z}\|_{\tilde{O}}+\left\|\bar{C} \bar{C}(.) \hat{y}_{2}^{0}\right\|_{\tilde{O}}+\left\|\bar{C} L(.) \bar{N} f\left(\bar{y}_{1}^{0}\right)\right\|_{\tilde{O}} \\
& \leq\|\bar{z}\|_{\tilde{O}}+c_{2}\left\|\hat{y}_{2}^{0}\right\|_{E}+c_{1}\left\|\bar{N} f\left(\bar{y}_{1}^{0}\right)\right\|_{L^{s}(0, T ; E)} \\
& \leq\|\bar{z}\|_{\tilde{O}}+c_{2}\left\|\hat{y}_{2}^{0}\right\|_{E}+c_{1} k\left(\left\|f\left(\bar{y}_{1}^{0}\right)\right\|, 0\right)\left\|f\left(\bar{y}_{1}^{0}\right)\right\|_{L^{r}\left(0, T ; E^{\alpha}\right)} \\
& \leq\|\bar{z}\|_{\tilde{O}}+c_{2}\left\|\hat{y}_{2}^{0}\right\|_{E}+c_{1} a_{2} \sup _{\theta \leq a_{2}} k(\theta, 0)
\end{aligned}
$$


Let's consider $\rho=\rho\left(a_{2}\right)=m-c_{2}\left\|\hat{y}_{2}^{0}\right\|_{E}-c_{1} a_{2} \sup _{\theta \leq a_{2}} k(\theta, 0)$

In order to have $\varphi_{1}\left(\bar{z}, \bar{y}_{1}^{0}\right) \in B(0, m)$, it suffices to consider $\|\bar{z}\|_{\tilde{O}} \leq \rho$.

For $\bar{z}_{1}, \bar{z}_{2} \in B(0, \rho)$, we have

$$
h\left(\bar{z}_{1}\right)-h\left(\bar{z}_{2}\right)=\bar{y}_{1,1}^{0}-\bar{y}_{1,2}^{0}=\varphi_{1}\left(\bar{z}_{1}, \bar{y}_{1,1}^{0}\right)-\varphi_{1}\left(\bar{z}_{2}, \bar{y}_{1,2}^{0}\right)=\varphi_{1}\left(\bar{z}_{1}, h\left(\bar{z}_{1}\right)\right)-\varphi_{1}\left(\bar{z}_{2}, h\left(\bar{z}_{2}\right)\right)
$$

which gives

$$
\begin{aligned}
\left\|h\left(\bar{z}_{1}\right)-h\left(\bar{z}_{2}\right)\right\|_{V} & \leq\left\|\varphi_{1}\left(\bar{z}_{1}, h\left(\bar{z}_{1}\right)\right)-\varphi_{1}\left(\bar{z}_{1},\left(\bar{z}_{2}\right)\right)\right\|_{V}+\left\|\varphi_{1}\left(\bar{z}_{1}, h\left(\bar{z}_{2}\right)\right)-\varphi_{1}\left(\bar{z}_{2}, h\left(\bar{z}_{2}\right)\right)\right\|_{V} \\
& \leq \alpha_{3}\left\|h\left(\bar{z}_{1}\right)-h\left(\bar{z}_{2}\right)\right\|_{V}+\left\|\bar{z}_{1}-\bar{z}_{2}\right\|_{\tilde{O}}
\end{aligned}
$$

then

$$
\left\|h\left(\bar{z}_{1}\right)-h\left(\bar{z}_{2}\right)\right\|_{V} \leq \frac{1}{1-\alpha_{3}}\left\|\bar{z}_{1}-\bar{z}_{2}\right\|_{\tilde{o}}
$$

which shows that $h$ is Lipschitzian.

\section{Numerical Approach}

\subsection{Numerical Approach}

We show the existence of a sequence of the initial gradient state and initial gradient speed which converges respectively to the regional initial gradient states and initial gradient speed to be observed in $\omega$.

Proposition 5.

We suppose that the hypothesis of Proposition 4 are verified, then for $\bar{Z} \in B(0, \rho)$, the sequence of the initial gradient condition defined in $B(0, m) \subset V$ by

$$
\left\{\begin{array}{l}
\tilde{y}_{1,0}^{0}=0 \\
\tilde{y}_{1, n+1}^{0}=\left(\bar{H}^{*}\right)^{\dagger}\left(\bar{z}-\bar{C} \bar{S}(.) \hat{y}_{2}^{0}-\bar{C} L(.) \bar{N} f\left(\tilde{y}_{1, n}^{0}\right)\right)
\end{array}\right.
$$

converges to $\bar{y}_{1}^{0}$ the regional initial gradient condition (the regional initial gradient state $y_{1}^{0}$ and the regional initial gradient speed $y_{1}^{1}$ ) to be observed in $\omega$, where $\hat{y}_{2}^{0}$ is the residual part of the initial gradient condition in $\Omega \backslash \omega$.

Proof

We have,

$$
\begin{aligned}
\left\|\tilde{y}_{1, n+1}^{0}-\tilde{y}_{1, n}^{0}\right\|_{V} & =\left\|\varphi_{1}\left(\bar{z}, \tilde{y}_{1, n+1}^{0}\right)-\varphi_{1}\left(\bar{z}, \tilde{y}_{1, n-1}^{0}\right)\right\|_{V} \\
& \leq \alpha_{3}\left\|\tilde{y}_{1, n}^{0}-\tilde{y}_{1, n-1}^{0}\right\|_{V} \leq \alpha_{3}^{2}\left\|\tilde{y}_{1, n-1}^{0}-\tilde{y}_{1, n-2}^{0}\right\|_{V} \\
& \leq \cdots \leq \alpha_{3}^{n}\left\|\tilde{y}_{1,1}^{0}-\tilde{y}_{1,0}^{0}\right\|_{V}=\alpha_{3}^{n}\left\|\tilde{y}_{1,1}^{0}\right\|_{V}
\end{aligned}
$$

or $\lim _{p \rightarrow+\infty} \alpha_{3}^{p}=0$, then there exists $n_{0} \in I N \quad \forall n \in I N, \quad \forall p \geq n_{0}$

$$
\begin{aligned}
\left\|\tilde{y}_{1, n+p}^{0}-\tilde{y}_{1, p}^{0}\right\|_{V} \leq & \left\|\tilde{y}_{1, n+p}^{0}-\tilde{y}_{1, n+p-1}^{0}\right\|_{V}+\left\|\tilde{y}_{1, n+p-1}^{0}-\tilde{y}_{1, n+p-2}^{0}\right\|_{V} \\
& +\left\|\tilde{y}_{1, n+p-2}^{0}-\tilde{y}_{1, n+p-3}^{0}\right\|+\cdots+\left\|\tilde{y}_{1, p+1}^{0}-\tilde{y}_{1, p}^{0}\right\|_{V} \\
& \leq \alpha_{3}^{n+p-1}\left\|\tilde{y}_{1,1}^{0}\right\|_{V}+\alpha_{3}^{n+p-2}\left\|\tilde{y}_{1,1}^{0}\right\|_{V}+\alpha_{3}^{n+p-3}\left\|\tilde{y}_{1,1}^{0}\right\|_{V}+\cdots+\alpha_{3}^{p}\left\|\tilde{y}_{1,1}^{0}\right\|_{V} \\
& =\alpha_{3}^{p}\left\|\tilde{y}_{1,1}^{0}\right\|_{V} \sum_{j=0}^{n-1} \alpha_{3}^{j}=\alpha_{3}^{p}\left\|\tilde{y}_{1,1}^{0}\right\|\left\|_{V}\left(\frac{1-\alpha_{3}^{n}}{1-\alpha_{3}}\right) \leq \alpha_{3}^{p}\right\| \tilde{y}_{1,1}^{0} \|_{V}\left(\frac{1}{1-\alpha_{3}}\right)
\end{aligned}
$$

Then $\left(\tilde{y}_{1, n}^{0}\right)_{n \geq 0}$ is a Cauchy sequence on $V$ and its convergence.

We consider $\bar{y}_{n}=f\left(\tilde{y}_{1, n}^{0}\right)$ and $\bar{z}_{n}=\bar{C} \bar{y}_{n}$ with 


$$
\left\{\begin{array}{l}
\bar{y}_{n}=\bar{S}(.) \bar{\nabla}^{*} \bar{\chi}_{\omega}^{*} \tilde{y}_{1, n}^{0}+\bar{S}(.) \hat{y}_{2}^{0}+L(.) \bar{N} f\left(\tilde{y}_{1, n}^{0}\right) \\
\bar{z}_{n}=\bar{H}^{*} \tilde{y}_{1, n}^{0}+\bar{C} \bar{S}(.) \hat{y}_{2}^{0}+\bar{C} L(.) \bar{N} f\left(\tilde{y}_{1, n}^{0}\right)
\end{array}\right.
$$

We have $\left(\bar{H}^{*}\right)^{\dagger}\left(\bar{z}-\bar{z}_{n}\right)=\tilde{y}_{1, n+1}^{0}-\tilde{y}_{1, n}^{0}$, then

$$
\bar{z}-\bar{z}_{n}=\bar{z}-\bar{C} \bar{S}(.) \hat{y}_{2}^{0}-\bar{C} L(.) \bar{N} f\left(\tilde{y}_{1, n}^{0}\right)-\bar{H}^{*} \tilde{y}_{1, n}^{0}=\bar{H}^{*}\left(\tilde{y}_{1, n+1}^{0}-\tilde{y}_{1, n}^{0}\right)
$$

then

$$
\left\|\bar{z}-\bar{z}_{n}\right\|_{\tilde{O}}=\left\|\tilde{y}_{1, n+1}^{0}-\tilde{y}_{1, n}^{0}\right\|_{V} \leq \alpha_{3}^{n}\left\|\tilde{y}_{1,1}^{0}\right\|_{V}
$$

which shows that the sequence $\bar{Z}_{n}$ converges to $\bar{Z}$ in $\tilde{O}$.

On the other hand, we have

$$
\left\|\bar{y}_{1}^{0}-\tilde{y}_{1, n}^{0}\right\|_{V}=\left\|h(\bar{z})-h\left(\bar{z}_{n}\right)\right\|_{V} \leq \frac{1}{1-\alpha_{3}}\left\|\bar{z}-\bar{z}_{n}\right\|_{\tilde{O}}
$$

Then $\tilde{y}_{1, n}^{0}$ converges to the regional initial gradient $\bar{y}_{1}^{0}$ to be observed in $\omega$.

\subsection{Algorithm}

Now let's consider the sequence $r_{n+1}=\bar{z}-\bar{C} \bar{S}(.) \hat{y}_{2}^{0}-\bar{C} L(.) \bar{N} f\left(\tilde{y}_{1, n}^{0}\right)$, then we have

$$
\tilde{y}_{1, n+1}^{0}=\left(\bar{H}^{*}\right)^{\dagger} r_{n+1} \text { and } \bar{z}_{n}=\bar{H}^{*} \tilde{y}_{1, n}^{0}+\bar{z}-r_{n+1}=r_{n}+\bar{z}-r_{n+1}
$$

Thus we obtain the following algorithm:

\section{Algorithm:}

1. Given the initial condition $\bar{y}_{1}^{0}=\left(y^{0}, y^{1}\right)$, the region $\omega$, The domain $D$ and the function of distribution $f$ and the accuracy threshold $\varepsilon, r_{1}=\bar{z}-\bar{C} \bar{S}(.) \hat{y}_{2}^{0}-\bar{C} L(.) \bar{N} f\left(\tilde{y}_{1,0}^{0}\right)$.

\section{Repeat}

a) $\tilde{y}_{1, n}^{0}=\left(\bar{H}^{*}\right)^{\dagger} r_{n}$

b) $\left\{\begin{array}{l}\frac{\partial \bar{y}_{n}(t)}{\partial t}=\overline{\mathcal{A}}_{n}(t)+\overline{\mathcal{N}} \bar{y}_{n}(t) \\ \bar{y}_{n}(0)=\bar{\nabla}^{*} \bar{\chi}_{\omega}^{*} \tilde{y}_{1, n}^{0}\end{array}\right.$

c) $\bar{z}_{n}=\bar{C}_{n}$

Until $\left\|\bar{z}-\bar{z}_{n}\right\|_{\tilde{O}} \leq \varepsilon$

3. $\tilde{y}_{1, n}^{0}$ which corresponds to the initial gradient condition to observed $\bar{y}_{1}^{0}$ in $\omega$.

Else $r_{n+1}=r_{n}+\bar{z}-r_{n+1}$ and go to step 2 .

\section{Conclusion}

The question of the regional gradient observability for semilinear hyperbolic systems was discussed and solved using sectorial approach, which uses sectorial properties of dynamical operators, the fixed point techniques and the properties of the linear part of the considered system. The obtained results are related to the considered subregion and the sensor location. Many questions remain open, such as the case of the regional boundary gradient observability of semilinear systems using Hilbert Uniqueness Method (HUM) and using the sectorial approach. These questions are still under consideration and the results will appear in a separate paper.

\section{References}

[1] El Jai, A., Simon, M.C. and Zerrik, E. (1993) Regional Observability and Sensor Structures. Sensors and Actuators Journal, 39, 95-102. http://dx.doi.org/10.1016/0924-4247(93)80204-T

[2] Zerrik, E., Bourray, H. and El Jai, A. (2004) Regional Observability for Semilinear Distributed Parabolic Systems. Journal of Dynamical and Control Systems, 10, 413-430. http://dx.doi.org/10.1023/B:JODS.0000034438.72863.ca 
[3] Boutoulout, A., Bourray, H., El Alaoui, F.Z. and Benhadid, S. (2014) Regional Observability for Distributed SemiLinear Hyperbolic Systems. International Journal of Control, 87, 898-910.

[4] Zerrik, E., Bourray, H. and Benhadid, S. (2007) Sensors and Regional Observability of the Wave Equation. Sensors and Actuators Journal, 138, 313-328. http://dx.doi.org/10.1016/j.sna.2007.05.017

[5] Boutoulout, A., Bourray, H. and El Alaoui, F.Z. (2013) Boundary Gradient Observability for Semilinear Parabolic Systems: Sectorial Approach. Mathematical Sciences Letters, 2, 45-54. http://dx.doi.org/10.12785/msl/020106

[6] Zeidler, E. (1990) Nonlinear Functional Analysis and Its Applications II/A Linear Applied Functional Analysis. Springer, Berlin.

[7] Boutoulout, A., Bourray, H. and Khazari, A. (2013) Gradient Observability for Hyperbolic System. International Review of Automatic Control (I.RE.A.CO), 6, 263-274.

[8] Boutoulout, A., Bourray, H. and El Alaoui, F.Z. (2012) Regional Gradient Observability for Distributed Semilinear Parabolic Systems. Journal of Dynamical and Control Systems, 18, 159-179. http://dx.doi.org/10.1007/s10883-012-9138-3

[9] Zuazua, E. (1993) Exact Controllability for Semilinear Wave Equations in One Space Dimension. Annalesde l’Institut Henri Poincaré: Analyse Non Linéaire, 10, 109-129.

[10] Zuazua, E. (1990) Exact Controllability for the Semilinear Wave Equations. Journal de Mathématiques Pures et Appliquées, 59, 1-31.

[11] Lions, J.L. (1988) Contrôlabilité Exacte, Perturbations et Stabilisation de Systèmes Distribués. Tome1, Masson, Paris.

[12] Lions, J.L. and Magenes, E. (1968) Problèmes aux limites non homogènes et applications. Vols. 1 et 2, Dunod, Paris.

[13] Henry, D. (1981) Geometric Theory of Semilinear Parabolic Systems. Lecture Notes in Mathematics 840, SpringerVerlag, Berlin Heidelberg, New York.

[14] Kassara, K. and El Jai, A. (1983) Algorithme pour la commande d'une classe de systèmes à paramètre répartis non linéaires. Revue. Marocaine. D’automatique. et de Traitement de Signal, 1, 3-24. 
Scientific Research Publishing (SCIRP) is one of the largest Open Access journal publishers. It is currently publishing more than 200 open access, online, peer-reviewed journals covering a wide range of academic disciplines. SCIRP serves the worldwide academic communities and contributes to the progress and application of science with its publication.

Other selected journals from SCIRP are listed as below. Submit your manuscript to us via either submit@scirp.org or Online Submission Portal.
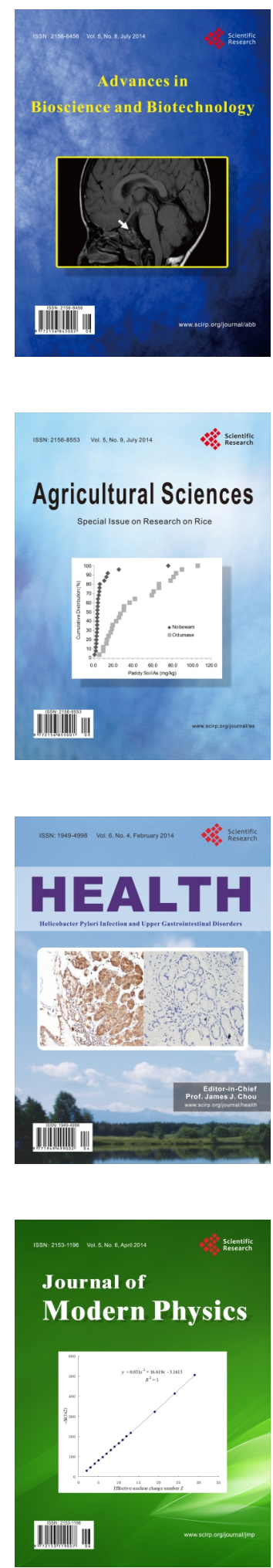
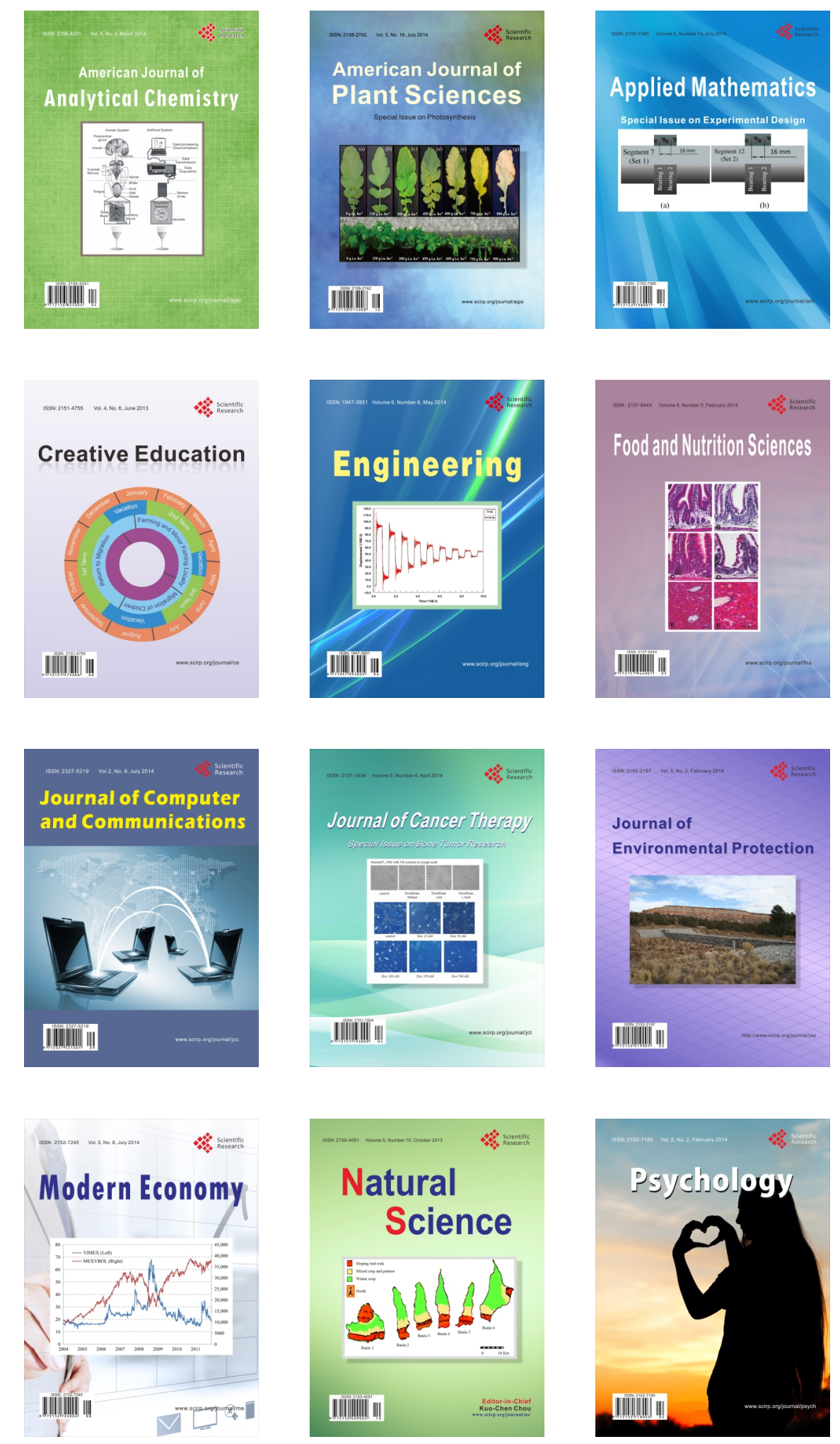